\title{
Linarin promotes osteogenic differentiation by activating the BMP-2/RUNX2 pathway via protein kinase A signaling
}

\author{
JIA LI $^{1}$, LINGYU HAO $^{1}$, JUNHUA WU $^{1}$, JIQUAN ZHANG $^{2}$ and JIANSHENG SU ${ }^{1}$ \\ ${ }^{1}$ Shanghai Engineering Research Center of Tooth Restoration and Regeneration, Department of Prosthodontics, \\ School of Stomatology, Tongji University, Shanghai 200072; ${ }^{2}$ Ministry of Education, Engineering Research Center \\ of Modern Preparation Technology of TCM, Shanghai University of Traditional Chinese Medicine, Shanghai 201203, P.R. China
}

Received August 30, 2015; Accepted February 2, 2016

DOI: $10.3892 /$ ijmm.2016.2490

\begin{abstract}
Linarin (LIN), a flavonoid which exerts both anti-inflammatory and antioxidative effects, has been found to promote osteogenic differentiation. However, the molecular mechanism of its effect on osteoblast differentiation was unclear. In the present study, LIN from Flos Chrysanthemi Indici $(\mathrm{FCI})$ was isolated in order to investigate the underlying mechanisms of LIN on MC3T3-E1 cells (a mouse osteoblastic cell line) and the osteoprotective effect of LIN in mice which had undergone an ovariectomy (OVX). The results revealed that LIN enhanced osteoblast proliferation and differentiation in MC3T3-E1 cells dose-dependently, with enhanced alkaline phosphatase (ALP) activity and mineralization of extracellular matrix. LIN upregulated osteogenesisrelated gene expression, including that of ALP, runt-related transcription factor 2 (RUNX2), osteocalcin (OCN), bone sialoprotein (BSP), and type I collagen (COL-I). Pretreatment with noggin, a bone morphogenetic protein-2 (BMP-2) antagonist, meant that LIN-induced gene expression levels of COL-1, ALP, OCN, BSP and RUNX2 were significantly reduced, as shown by RT-qPCR. Western blot analysis showed that LIN dose-dependently increased the protein levels of
\end{abstract}

Correspondence to: Dr Jiansheng Su, Shanghai Engineering Research Center of Tooth Restoration and Regeneration, Department of Prosthodontics, School of Stomatology, Tongji University, Middle Yanchang Road 399, Shanghai 200072, P.R. China

E-mail: sjs@tongji.edu.cn

Abbreviations: LIN, linarin; OVX, ovariectomy; ALP, alkaline phosphatase; OCN, osteocalcin; BSP, bone sialoprotein; COL-I, type I collagen; BMP-2, bone morphogenetic protein-2; PKA, protein kinase A; BMPs, bone morphogenetic proteins; RUNX2, runt-related transcription factor 2; FCI, Flos Chrysanthemi Indici; CCK-8, Cell Counting Kit-8; DMSO, dimethyl sulfoxide; BV/TV, bone volume/ tissue volume; BS/TV, bone surface/tissue volume; Tb.Sp, trabecular space; Tb.N, trabecular number; BMD, bone mineral density

Key words: bone morphogenetic protein-2, linarin, osteoblast differentiation, osteoporosis, protein kinase A
BMP-2 and RUNX2 and enhanced the phosphorylation of SMAD1/5. In addition, LIN dose-dependently upregulated protein kinase A (PKA) expression. H-89 (a PKA inhibitor) partially blocked the LIN-induced protein increase in BMP-2, p-SMAD1/5 and RUNX2. We noted that LIN preserved the trabecular bone microarchitecture of ovariectomized mice in vivo. Moreover, pretreatment with LIN significantly lowered serum levels of ALP and OCN in ovariectomized mice. Our data indicated that LIN induced the osteogenic differentiation and mineralization of MC3T3-E1 osteoblastic cells by activating the BMP-2/RUNX2 pathway through PKA signaling in vitro and protected against OVX-induced bone loss in vivo. The results strongly suggest that LIN is a useful natural alternative for the management of postmenopausal osteoporosis.

\section{Introduction}

Osteoporosis is a bone disease which is characterized by decreased bone strength, and an imbalance between bone formation and bone resorption causes osteoporosis $(1,2)$. Currently, there are several therapeutic options available for managing osteoporosis. The bone resorption inhibitors (e.g., bisphosphonates, calcitonin and estrogens) are widely used in the treatment of osteoporosis (3). The bone resorption inhibitors regulate bone density by blocking osteoclast function; however, long-term use of these drugs may have severe side effects. For instance, the prolonged use of estrogen increases the risk of breast cancer and heart disease (4). Moreover, the efficacy of bone resorption inhibitors in recovering bone mass is relatively moderate. Hence, natural compounds or other synthetic substances for bone formation with fewer undesirable side effects are an alternative strategy for managing osteoporosis.

Bone formation is comprised of a complex series of events during which mesenchymal stem cells are differentiated into osteoblasts. In osteoblast differentiation, bone morphogenetic proteins (BMPs) play an essential role in bone formation via the production of bone specific matrix proteins $(5,6)$. BMP-2, an important growth factor in the BMP subfamily, modulates osteoblast differentiation by stimulating osteoblast-related transcriptional factors, including runt-related transcription factor 2 (RUNX2) and Osterix (7,8). Protein kinase A (PKA, 
a serine/threonine kinase) regulates many cellular functions, including immune response, sugar metabolism, and osteoblast differentiation (9-11). Previous studies have indicated that several osteoblast-specific transcriptional factors are regulated by PKA (11-13). Although the precise mechanisms are not yet fully understood, it has been noted that PKA acts downstream of the BMP-2/RUNX2 pathway and enhances osteogenic differentiation (11).

Flos Chrysanthemi Indici (FCI), the flower of Chrysanthemum indicum L., is a commonly used herb in traditional Chinese medicine, and it has effective antimicrobial, antioxidative, and antimycotic properties (14-17). Linarin (LIN), a natural flavonoid compound in FCI, has been shown to exert various pharmacological effects, including anti-inflammatory, neuroprotective, cardioprotective and antioxidative effects (18-22). LIN has previously been shown to induce the differentiation and mineralization of the mouse osteoblastic cell line MC3T3-E1 (18); however, the mechanism of action has not yet been revealed. The present study aimed to investigate the effects of LIN on the differentiation and mineralization of MC3T3-E1 osteoblastic cells and the involvement of the PKA-mediated BMP-2/RUNX2 signaling pathway. The effect of LIN on preventing bone loss was studied using an ovariectomized mice model.

\section{Materials and methods}

Plant materials and reagents. The FCI was bought from the Haozhou medicinal material market (Anhui, China). The water was purified using an RU-B laboratory ultrapure water system (Shanghai Tauto Biotech Co., Ltd., Shanghai, China). The Cell Counting Kit-8 (CCK-8) was bought from Dojindo Molecular Technologies, Inc. (Tokyo, Japan). The alkaline phosphatase (ALP) activity assay kit and bicinchoninic acid (BCA)-protein assay kit were both obtained from Beyotime Institute of Biotechnology (Jiangsu, China). Noggin and H-89 dihydrochloride hydrate were purchased from Sigma-Aldrich (St. Louis, MO, USA). Primary antibodies targeting p-SMAD1/5 (Ser463/465; \#9516) and SMAD1/5 (\#12656) were obtained from Cell Signaling Technology, Inc. (Danvers, MA, USA). Primary antibodies targeting PKA (ab75991), BMP-2 (ab14933), RUNX2 (ab76956) and $\beta$-actin (ab32572) were purchased from Abcam (Cambridge, UK).

Preparation of LIN from FCI. The FCI was extracted three times with $80 \%$ ethanol (each extraction was for $2 \mathrm{~h}$ ). The extracts were filtered, combined, and evaporated to dry under reduced pressure at $55^{\circ} \mathrm{C}$. The dried product of the ethanolic extract was dissolved in purified water, and extracted successively with n-hexane, ethyl acetate and n-butanol. The ethyl acetate fraction was subjected to high-speed countercurrent chromatography (HSCCC; Shanghai Tauto Biotech Co., Ltd.) with a two-phase solvent system [chloroform/methanol/water $(4: 3: 2, \mathrm{v} / \mathrm{v} / \mathrm{v})]$. Under optimized conditions, LIN was isolated and purified by HSCCC (Fig. 1B). Finally, the structure of LIN was identified by proton and carbon-13 nuclear magnetic resonance (NMR) spectra (Varian Unity Inova 500 NMR system) (Varian Medical Systems, Inc., Palo Alto, CA, USA), as previously described (23). The purity of LIN (98.5\%) was then determined by high-performance liquid chromatography (Dionex Corp., Sunnyvale, CA, USA) (Fig. 1C).
LIN powder was dissolved in vehicle (10\% Tween-80 in physiological saline) to provide a final concentration of 5 and $15 \mathrm{mg} / \mathrm{ml}$ for in vivo experiments. For in vitro experiments, LIN was dissolved in dimethyl sulfoxide (DMSO) (final DMSO concentration in the culture was less than $0.5 \%$ ).

Cell culture and osteoblast differentiation. The MC3T3-E1 cell line (a mouse osteoblast cell line) was obtained from the American Type Culture Collection (ATCC; Manassas, VA, USA). Cells were cultured in $\alpha$-modified minimal essential medium ( $\alpha$-MEM; Invitrogen, Carlsbad, CA, USA) at $37^{\circ} \mathrm{C}$ in an atmosphere with $5 \%$ carbon dioxide. Subsequently, $10 \%$ fetal bovine serum, $1 \%$ penicillin, and $1 \%$ streptomycin were added to the medium. To induce osteogenic differentiation, cells were cultured until they reached confluence and transferred to $\alpha$-MEM containing $10 \%$ FBS, $1 \%$ penicillin, $1 \%$ streptomycin, $10 \mathrm{mM} \beta$-glycerophosphate and $50 \mu \mathrm{g} / \mathrm{ml}$ ascorbic acid.

Cell proliferation assay. The cell proliferation rate was determined using CCK-8. In this assay, MC3T3-E1 cells were cultured in 96-well plates $\left(4 \times 10^{3}\right.$ cells/well) for $24 \mathrm{~h}$. The cells in various wells were then treated with $0,10^{-9}, 10^{-8}, 10^{-7}, 10^{-6}$ or $10^{-5} \mathrm{M}$ LIN concentrations. After 48 and $72 \mathrm{~h}$ incubation, $10 \mu \mathrm{l} \mathrm{CCK}-8$ reagent was added to each well and cultured for $1 \mathrm{~h}$. Absorbance was then measured at $450 \mathrm{~nm}$ using a microplate reader (Tecan, Grödig, Austria). All tests were performed in triplicate.

Measuring ALP activity. MC3T3-E1 cells were incubated with or without LIN in 24-well plates. After 1,3,5 and 7 days of incubation, cells were washed two times with phosphate-buffered saline (PBS) and lysed in $0.2 \%$ Triton X-100. The lysate was then centrifuged at $10,000 \mathrm{x}$ g for $5 \mathrm{~min}$. The supernatant was collected and incubated in p-Nitrophenyl-phosphate (pNPP) at $37^{\circ} \mathrm{C}$ for $30 \mathrm{~min}$. After the reaction was stopped, absorbance was measured at $405 \mathrm{~nm}$. The protein levels were measured using a BCA protein assay kit, as previously described (24). Absorbance was measured at $560 \mathrm{~nm}$. ALP activity was normalized to an absorbance value at $405 \mathrm{~nm} / \mathrm{mg}$ of total protein.

Determination of mineralized matrix. Mineralization in the MC3T3-E1 cells was determined by staining with Alizarin red $\mathrm{S}$, which selectively binds to calcium and yields a dark red stain. The cells $\left(2 \times 10^{5}\right)$ were cultured in 24 -well plates with various doses of LIN for 21 days. Staining with $40 \mathrm{mM}$ Alizarin red $\mathrm{S}$ ( $\mathrm{pH} 4.2$ ) was performed, and the images of calcified matrices were photographed under a Nikon microscope. To quantify the calcified matrix, Alizarin red S stained cells were treated with $100 \mathrm{mM}$ cetylpyridinium chloride for $1 \mathrm{~h}$. The absorbance was then measured at $570 \mathrm{~nm}$ on enzyme-linked immunosorbent assay (ELISA) reader.

Real time-quantitative polymerase chain reaction ( $R T-q P C R)$. MC3T3-E1 cells $\left(1 \times 10^{5}\right)$ were incubated with or without LIN in 24-well plates for 7 days. Total ribonucleic acid (RNA) was extracted using RNeasy Mini kits (Qiagen, Inc., Valencia, CA, USA). In addition, the complementary deoxyribonucleic acid (cDNA) was synthesized from $1 \mu \mathrm{g}$ total RNA of each sample using reverse transcription kits (Takara Bio, Inc., Otsu, Japan). RT-qPCR was performed on an ABI 7500 Sequencing 
A<smiles>COc1ccc(-c2cc(=O)c3c(O)cc(OC4OC(OC5OC(C)C(O)C(O)C5O)C(O)C(O)C4O)cc3o2)cc1</smiles>
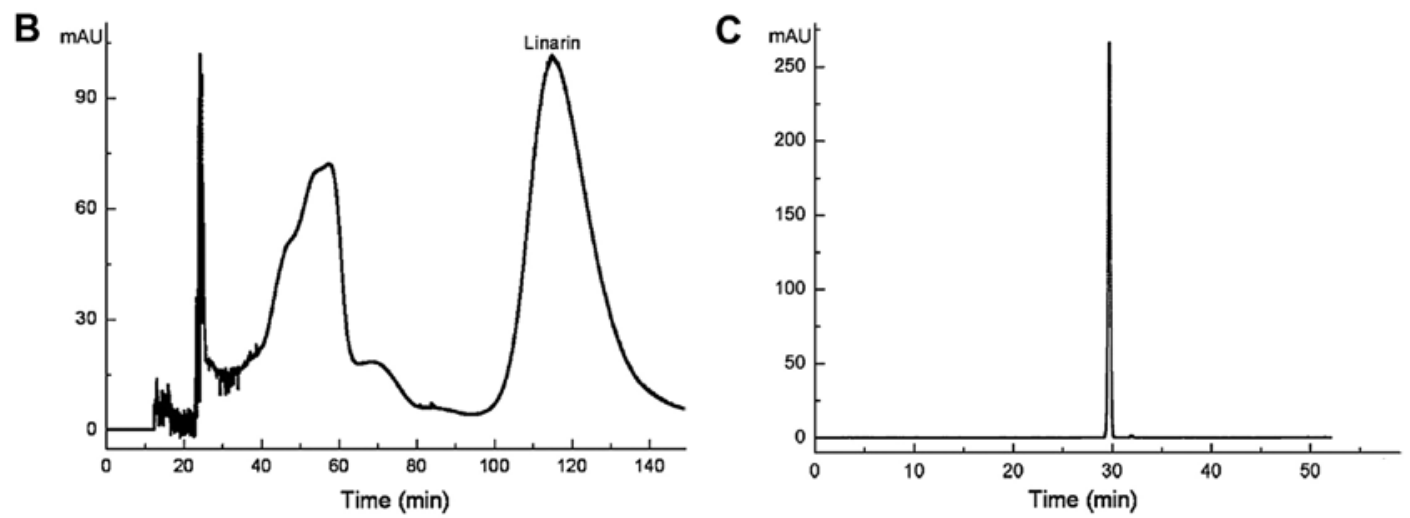

Figure 1. Preparation and purification of linarin (LIN) from Flos Chrysanthemi Indici (FCI). (A) Chemical structure of LIN. (B) High-speed countercurrent chromatography (HSCCC) chromatogram of LIN from ethyl acetate fraction of FCI. (C) HPLC chromatogram of LIN purified by HSCCC.

Table I. Specific primers used for RT-qPCR.

\begin{tabular}{lll}
\hline Gene & \multicolumn{1}{c}{ Forward } & \multicolumn{1}{c}{ Reverse } \\
\hline COL-I & 5'-GAGCGGAGTACTGGATCG-3' & 5'-GCTTCTTTTCCTTGGGGTT-3' \\
ALP & 5'-GATCATTCCCACGTTTTCACATT-3' & 5'-TTCACCGTCCACCACCTTGT-3' \\
OCN & 5'-GAGGACCATCTTTCTGCTCACTCT-3' & 5'-TTATTGCCCTCCTGCTTGGA-3' \\
BSP & 5'-AGGACTGCCGAAAGGAAGGTTA-3' & 5'-AGTAGCGTGGCCGGTACTTAAA-3' \\
RUNX2 & 5'-GCACAAACATGGCCAGATTCA-3' & 5'-AAGCCATGGTGCCCGTTAG-3' \\
$\beta$-actin & 5'-TCTGCTGGAAGGTGGACAGT-3' & 5'-CCTCTATGCCAACACAGTGC-3' \\
\hline
\end{tabular}

COL-I, type I collagen; ALP, alkaline phosphatase; OCN, osteocalcin; BSP, bone sialoprotein; RUNX2, runt-related transcription factor 2.

Detection system (Applied Biosystems, Foster City, CA, USA) using SYBR Premix Ex Taq kits (Takara Bio, Inc.). The PCR conditions were as follows: $50^{\circ} \mathrm{C}$ incubation for $2 \mathrm{~min}, 95^{\circ} \mathrm{C}$ initial denaturation for $10 \mathrm{~min}$, then $95^{\circ} \mathrm{C}$ for $15 \mathrm{sec}, 60^{\circ} \mathrm{C}$ for $30 \mathrm{sec}$ and $72^{\circ} \mathrm{C}$ for $30 \mathrm{sec}$ for 40 cycles. Fluorescence data were collected in the extension step $\left(72^{\circ} \mathrm{C}\right.$ for $\left.30 \mathrm{sec}\right)$. All reactions were run in triplicate. PCR data were analyzed using ABI Prism 7000 SDS software, and the housekeeping gene $\beta$-actin served as an internal control. The target primer sequences are listed in Table I.

Western blot analysis. MC3T3-E1 cells were incubated with or without LIN in 6-well plates for different time intervals. After washing with PBS twice, cells were incubated in lysis buffer [pH 7.9; 10 mM 4-(2-hydroxyethyl)-1-piperazineethanesulfonic acid, $10 \mathrm{mM}$ potassium chloride, $0.1 \mathrm{mM}$ ethylenediaminetetraacetic acid (EDTA), $1 \mathrm{mM}$ dithiothreitol, and protease inhibitors leupeptin $(10 \mu \mathrm{g} / \mathrm{ml})$, aprotinin $(10 \mu \mathrm{g} / \mathrm{ml})$ and $0.1 \mathrm{mM}$ phenylmethylsulfonyl fluoride] for $30 \mathrm{~min}$ at $4^{\circ} \mathrm{C}$. For western blot analysis, the protein lysate $(30 \mu \mathrm{g})$ was subjected to sodium dodecyl sulfate-polyacrylamide gel electrophoresis (10\% gels) and transferred to polyvinylidene difluoride membranes (Pall Corporation, Port Washington, NY, USA). After blocking nonspecific interactions with TBS buffer $(0.05 \%$ Tween-20 and $5 \%$ non-fat milk) for $1 \mathrm{~h}$, the membranes were incubated with target primary antibodies (BMP-2, SMAD1/5, p-SMAD1/5, RUNX2, PKA and $\beta$-actin at 1/1,000 dilution). Subsequently, the membranes were washed three times with washing buffer containing $0.05 \%$ Tween-20 and incubated with appropriate horseradish peroxidase-conjugated secondary antibody (sc-2005; Santa Cruz Biotechnology, Inc., Santa Cruz, CA, USA) at 1:5,000 dilution. The membranes were washed again, and target bands were detected with an enhanced chemiluminescence detection system (Santa Cruz Biotechnology, Inc.). $\beta$-actin was used as the internal control. The experiments were replicated three times.

Animals. Female C57/BL6 mice $(21 \pm 1.5$ g, 8 weeks old) were purchased from the Shanghai Laboratory Animal Center (SLAC), Shanghai, China. The mice were housed in the Laboratory Animal Facility at Tongji University under 
controlled temperature $\left(22-24^{\circ} \mathrm{C}\right)$ and humid $(50-60 \%)$ conditions and exposed to a 12-h light/dark cycle with free access to food (SLAC standard mice food) and water. The mice were acclimatized for at least seven days before the experiments. All animal experiments were performed according to the National Institutes of Health Guidelines for the Care and Use of Laboratory Animals and were approved by the Animal Care and Use Committee of Shanghai Tongji University, Shanghai, China.

Ovariectomy (OVX)-induced osteoporosis. The mice were randomly divided into four groups ( $\mathrm{n}=10 /$ group), namely three groups which underwent an OVX and one sham-operated group (control group). Animals in the OVX groups were ovariectomized bilaterally under sodium pentobarbital anesthesia (intraperitoneal dose of $40 \mathrm{mg} / \mathrm{kg}$ ), whereas control group mice were sham-operated for comparison. After 1 week of recovery from surgery, the mice which underwent an OVX were treated with either vehicle (10\% Tween-80) or LIN (daily intragastric administration of LIN at 50 and $150 \mathrm{mg} / \mathrm{kg}$ body weight, respectively, in the low, LIN-L, and high, LIN-H, LIN-treated groups) for 8 weeks. The dosages of LIN were safe for mice, as has been previously described (22). The control group mice were administered orally with vehicle. At the end of the 8 -week treatment, the mice were sacrificed by cardiac puncture and the blood was collected. The femurs were excised and then immediately fixed in $4 \%$ paraformaldehyde for further experiments. Serum ALP and osteocalcin (OCN) levels were measured using mouse-specific ELISA kits (Biomedical Technologies, Inc., Stoughton, MA, USA).

The left-side femurs of the mice were scanned with a cone-beam micro-computed tomography (CT) system (SkyScan 1076; SkyScan, Kontich, Belgium) at a resolution of $18 \mu \mathrm{m}$ using the following settings: X-ray voltage, $40 \mathrm{kV}$; electric current, $250 \mu \mathrm{A}$; and rotation step, $0.6^{\circ}$. Bone parameters of femurs were analyzed directly from the original three-dimensional images, and micro-CT analysis was performed as previously described (25). Microstructural indices of the trabecular bone of the femurs including bone volume/tissue volume (BV/TV), bone surface (BS/TV), trabecular space (Tb.Sp), trabecular number (Tb.N), as well as bone mineral density (BMD) were determined. Tissues were removed, fixed in $4 \%$ paraformaldehyde for one day at $4{ }^{\circ} \mathrm{C}$, and then decalcified in $10 \%$ EDTA. Decalcified bones were embedded in paraffin, sectioned, and stained with haematoxylin and eosin $(\mathrm{H} \& \mathrm{E})$ for histological examination.

Statistical analysis. Data are expressed as the means \pm standard deviation. One-way analysis of variance was used to compare the results between different groups. A P-value $<0.05$ was considered to indicate a statistically significant difference.

\section{Results}

Effect of LIN on the proliferation of MC3T3-E1 cells. The effect of LIN $\left(10^{-9}, 10^{-8}, 10^{-7}, 10^{-6}\right.$ and $\left.10^{-5} \mathrm{M}\right)$ on osteoblastic cell viability was examined, and the results demonstrated that LIN dose-dependently increased cell proliferation (Fig. 2). Based on the above results, $0.1,1$ and $10 \mu \mathrm{M}$ of LIN were used in the following experiments.

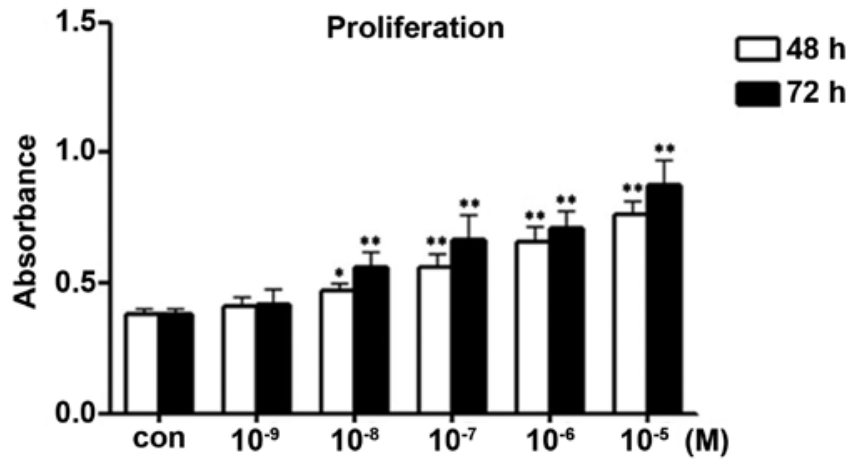

Figure 2. Effect of linarin (LIN) on cell proliferation of MC3T3-E1 cells. The cells were treated with different concentrations (M) of LIN for 2 and 3 days respectively, and cell viability was measured by CCK- 8 assay. Administration of LIN increased the proliferation of MC3T3-E1 cells. Data are represented as the means \pm SD of three independent experiments. ${ }^{*} \mathrm{P}<0.05$ as compared with control; ${ }^{* *} \mathrm{P}<0.01$ as compared with control.

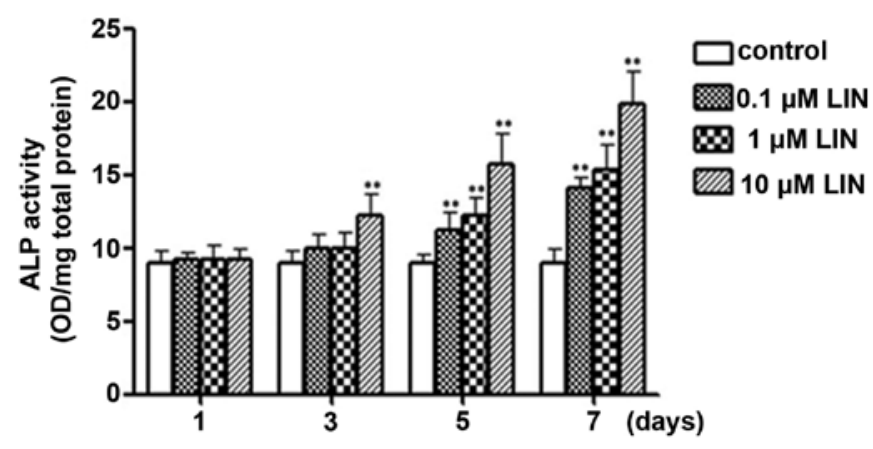

Figure 3. Effect of linarin (LIN) on alkaline phosphatase (ALP) activity of MC3T3-E1 cells. After treatment with various cocentrations of LIN for various time intervals, ALP activity was assessed using a commercial ALP kit. Administration of LIN increased the differentiation of MC3T3-E1 cells. Data are represented as the means \pm SD of three independent experiments. ${ }^{* * *} \mathrm{P}<0.01$ as compared with control.

LIN enhances ALP activity of MC3T3-E1 cells. ALP is a key enzyme for the early differentiation of osteoblasts (26). Hence, the effect of LIN on ALP activity was assessed. The results demonstrated that ALP activity was not significantly increased by LIN after 1 and 3 days of incubation, whereas ALP activity was increased by LIN in a dose-dependent manner at 5 and 7 days after treatment (Fig. 3).

LIN enhances the mineralization of the extracellular matrix in MC3T3-E1 cells. The development of calcified nodules is an important marker of osteoblast maturation. When compared to the control, LIN (1 and $10 \mu \mathrm{M})$ significantly enhanced the formation of calcified nodules in MC3T3-E1 cells after 21 days of incubation. The calcified nodules appeared bright red in color after Alizarin red S staining (Fig. 4). The results suggest that LIN promotes the mineralization of extracellular matrix in a dose-dependent manner in MC3T3-E1 cells.

BMP-2 mediates LIN-induced upregulation of osteogenesisrelated gene expression in MC3T3-E1 cells. The expression of osteogenic gene markers ALP, RUNX2, OCN, bone sialoprotein (BSP), and type I collagen (COL-I) in MC3T3-E1 cells was examined using RT-qPCR after incubation with $10 \mu \mathrm{M}$ 


\section{A $\quad \operatorname{LIN}(\mu \mathrm{M})$}
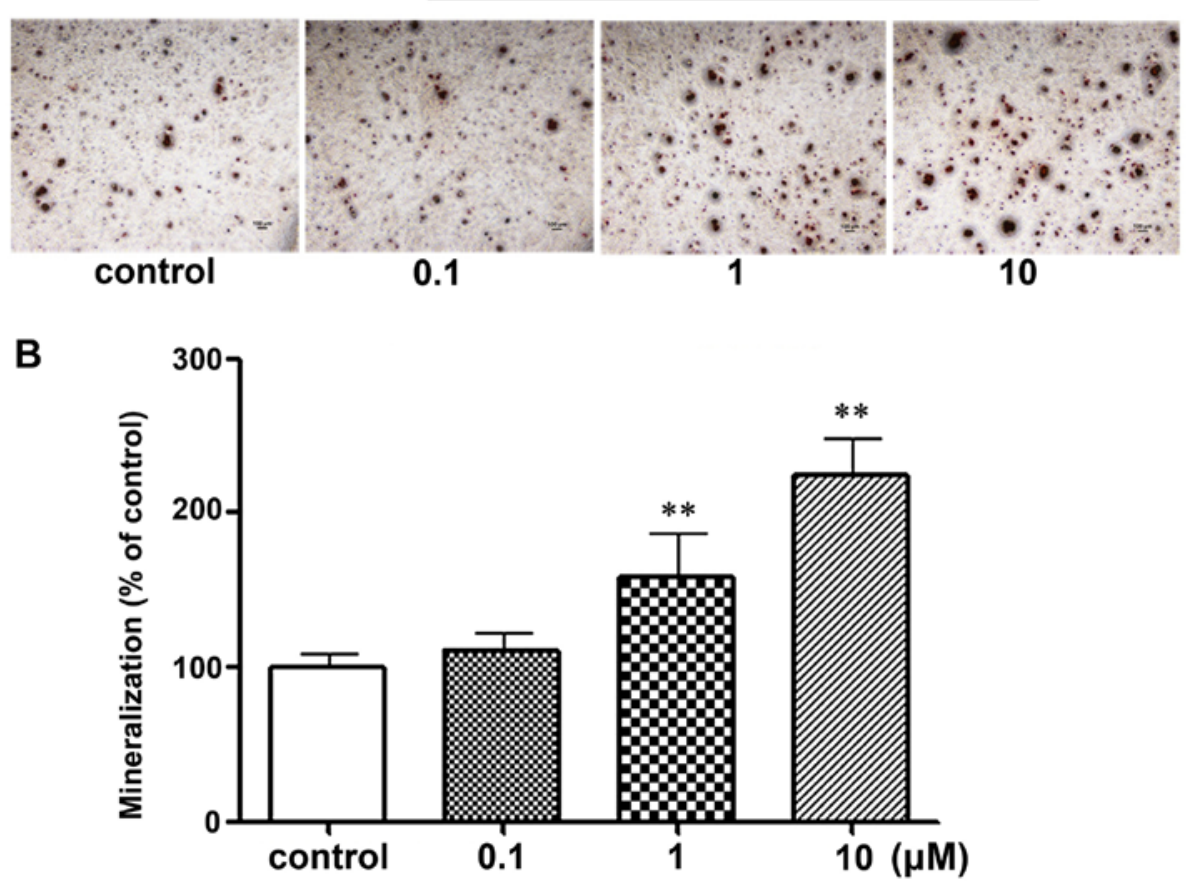

Figure 4. Effect of linarin (LIN) on mineralization of MC3T3-E1 cells. (A) After treatment with various concentrations of LIN for 21 days, the mineral nodule formation was assessed by Alizarin red S dye (x4). Scale bar, $100 \mu \mathrm{m}$. (B) The bound, stained cells were washed with cetylpyridinium chloride and quantified using a Bio-Rad ELISA reader. LIN increased the mineralization of MC3T3-E1 cells. Data are represented as the means \pm SD of three independent experiments. ${ }^{* *} \mathrm{P}<0.01$ as compared with control.

LIN for 7 days. The results demonstrated that the osteogenesisrelated gene expression levels were significantly increased in MC3T3-E1 cells treated with LIN (Fig. 5A).

It has previously been noted that BMP-2 plays a critical role in bone formation and remodeling and noggin is a specific inhibitor for BMP-2 signaling pathway (27). To further reveal the role which BMP-2 plays in LIN-induced osteogenesis, MC3T3-E1 cells were pretreated with noggin $(100 \mathrm{ng} / \mathrm{ml})$ for $2 \mathrm{~h}$. Pretreatment with noggin significantly reduced the LIN-induced gene expression of COL-1, ALP, OCN, BSP and RUNX2 (Fig. 5A). This result indicates that LIN-induced upregulation of osteogenesis-related gene expressions is activated via the BMP-2-dependent pathway.

LIN stimulates osteoblast differentiation via the BMP-2 signaling pathway. The downstream transcription factors SMAD and RUNX2 are known to mediate the action of BMP-2 (28). To further examine whether the BMP-2 signaling pathway was influenced by LIN, the expression of BMP-2, p-SMAD1/5, and RUNX2 in MC3T3-E1 cells after treatment with LIN $(0.1-10 \mu \mathrm{M})$ was determined using western blot analysis. The results showed that LIN dose-dependently increased BMP-2 and RUNX2 protein levels (Fig. 5B). LIN had little impact on unphosphorylated SMAD1/5 expression; however, it increased p-SMAD1/5 protein levels (Fig. 5B). These results suggest that LIN promotes the osteoblast differentiation via the BMP-2/SMAD-dependent RUNX2 signaling pathway.

LIN-induced osteoblast differentiation is mediated by PKA activation. To determine whether PKA was associated with LIN-induced osteoblast differentiation, PKA protein expres- sion levels after LIN treatment were analyzed by western blot analysis. LIN markedly stimulated PKA protein expression in a time- and dose-dependent manner (Fig. 6A and B). Pretreatment with H-89 (PKA inhibitor, $2 \mu \mathrm{M}$ ) partially blocked the LIN-induced increase in BMP-2, p-SMAD1/5 and RUNX2 protein accumulation (Fig. 6C). These results suggest that LIN promoted osteoblast differentiation through the PKA-dependent pathways, with associated BMP-2/RUNX2 activation.

Effects of LIN on OVX-induced bone loss. To examine the in vivo effect of LIN on OVX-induced bone loss, the trabecular bone microarchitecture of ovariectomized mice was examined using micro-CT. The micro-CT scan revealed that the OVX induced extensive bone loss at metaphyseal distal femurs of mice; quantitative analysis of bone parameters revealed a significant reduction of BV/TV, BS/TV and Tb.N and an increase of Tb.Sp, suggesting osteolysis. LIN dose-dependently preserved the trabecular bone microarchitecture of ovariectomized mice (Fig. 7A). Moreover, LIN slightly increased BMD compared to the control OVX group; however, this increase was not statistically significant. In addition, histological analysis verified the protective role of LIN on OVX-induced bone loss (Fig. 7A). Finally, an OVX resulted in an increase of serum ALP and OCN, whereas LIN pretreatment $(150 \mathrm{mg} / \mathrm{kg}$ for 8 weeks) had significantly decreased OVX-induced serum ALP and OCN levels (Fig. 7C).

\section{Discussion}

It has previously been shown that osteoblasts play a fundamental role in bone formation and remodeling; however, these failed to produce bone regrowth in musculoskeletal 
A

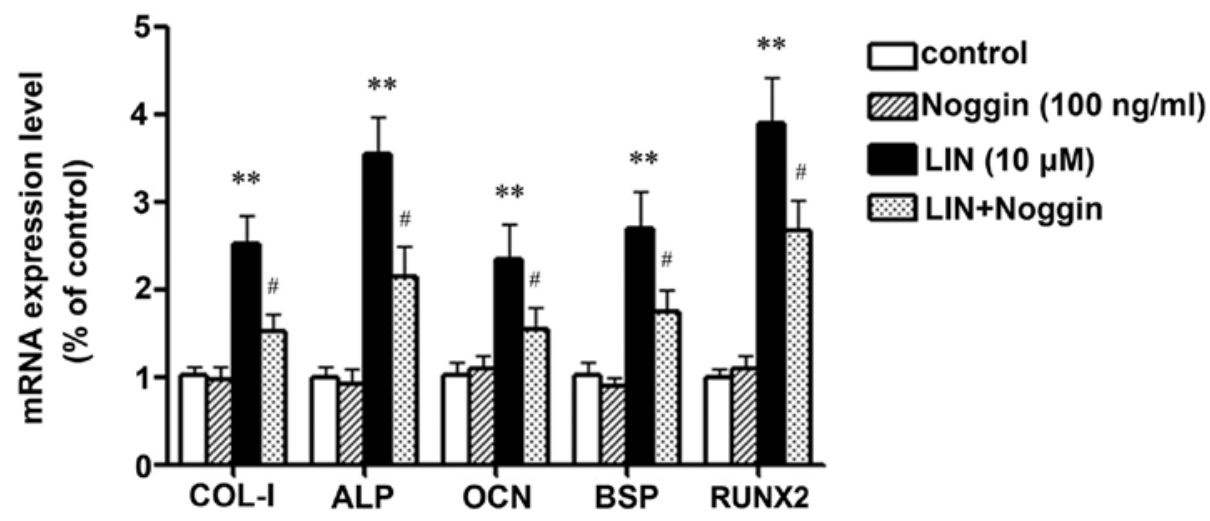

B
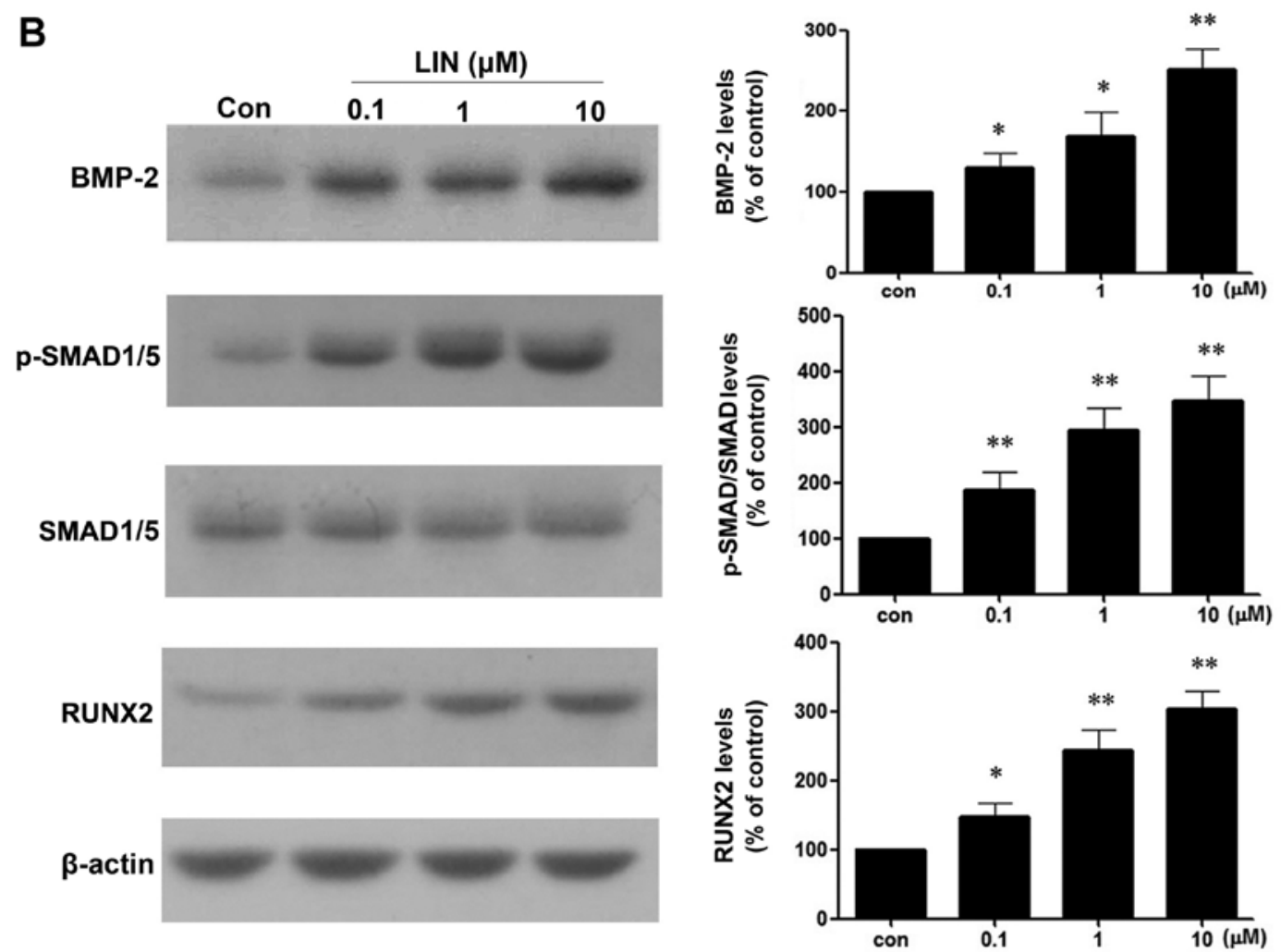

Figure 5. Linarin (LIN) promotes osteoblast differentiation through the bone morphogenetic protein-2 (BMP-2) signaling pathway. (A) MC3T3-E1 cells were pretreated with $10 \mu \mathrm{M}$ LIN or vehicle (DMSO) in the presence or absence of $100 \mathrm{ng} / \mathrm{ml}$ noggin and were then induced to undergo osteogenesis for 7 days. Levels of type I collagen (COL-I), alkaline phosphatase (ALP), osteocalcin (OCN), bone sialoprotein (BSP) and runt-related transcription factor 2 (RUNX2) mRNA expression were analyzed by RT-qPCR. (B) Western blot analysis of BMP-2, SMAD1/5 phosphorylation and RUNX2 expression after treatment of MC3T3-E1 cells with the indicated concentration of LIN for $48 \mathrm{~h}$. The quantitation of the blots was undertaken by densitometric analysis. Data are represented as the means \pm SD of three independent experiments. ${ }^{*} \mathrm{P}<0.05,{ }^{*} \mathrm{P}<0.01$ compared with the control; ${ }^{\#} \mathrm{P}<0.01$ compared with LIN.

disorders such as osteoporosis $(1,29)$. Recently, it has been suggested that natural flavonoid compounds exert a therapeutic effect on osteoporosis (30). Hence, in the present study, the effects of LIN, a natural flavonoid compound isolated from Chrysanthemum indicum, on osteoblastic MC3T3-E1 cells in vitro and OVX-induced bone loss in vivo were assessed. When compared with the control, LIN significantly promoted the growth of MC3T3-E1 cells in a dose-dependent manner. Osteogenic differentiation is a complex process which results in the formation of mineralized extracellular matrix. There are large number of regulators governing the osteogenic differentiation such as ALP, COL-I, OCN and BSP, and ALP is a key enzyme that provides a high concentration of phosphate at an earlier stage of mineral deposition (26). COL-I, an important component of the bone extracellular matrix, connects the cell surface integrins with other extracellular matrix proteins (31). BSP is localized in the extracellular matrix, which can increase the nucleation of hydroxyapatite crystal formation and promote the calcium absorption and mineralization (32). OCN is a protein abundantly found in bone. In the present study, we noted that LIN significantly increased the mRNA gene expression of ALP and upregulated the mRNA levels of BSP, COL-1 and OCN, and thereby enhanced mineralization. Thus, LIN promoted cell differentiation and the maturation of osteoblasts, indicating that LIN is beneficial in preventing osteoporosis by promoting osteoblast function.

To further understand the effect of LIN on MC3T3-E1 cell differentiation, the signaling pathways responsible for 
A

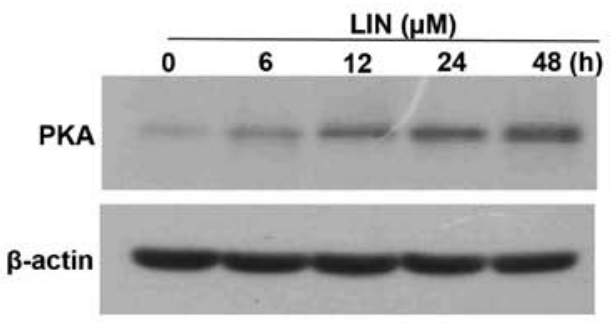

B

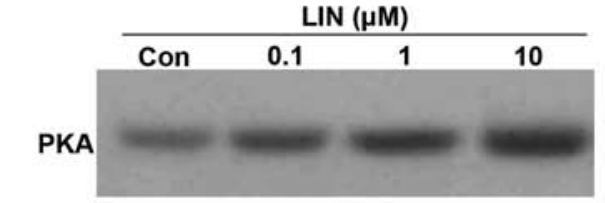

$\beta$-actin

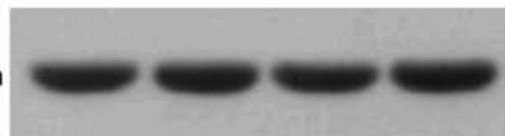

C
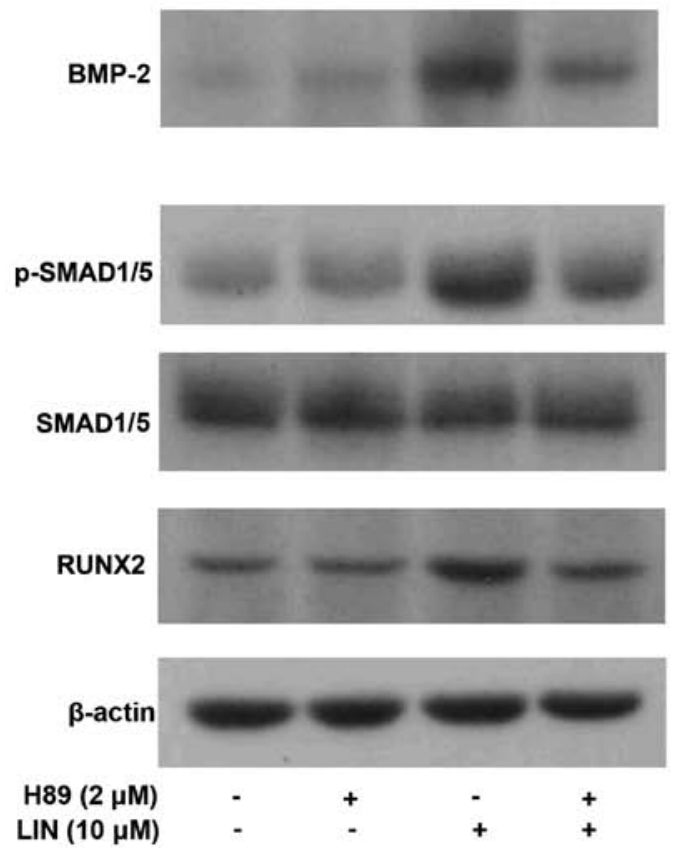
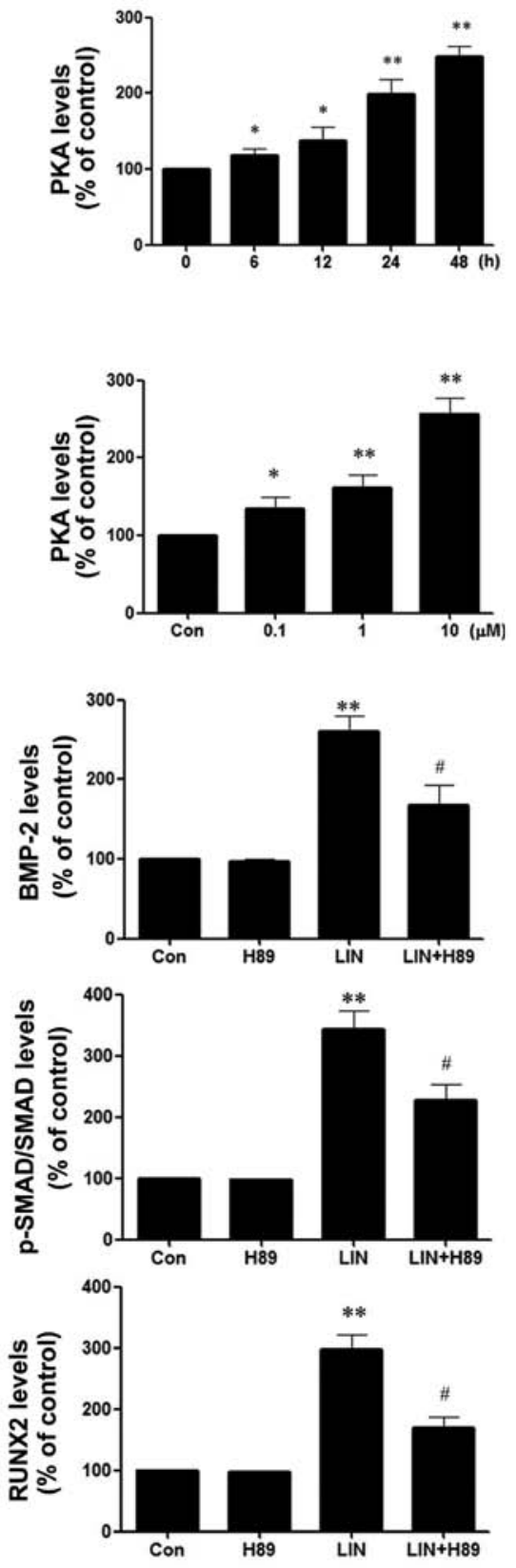

Figure 6. Role of protein kinase A (PKA) in the upregulation of osteoblastic activity by linarin (LIN). (A) Western blot analysis of PKA expressions after treatment with $10 \mu \mathrm{M}$ LIN for 6,12, 24 or $48 \mathrm{~h}$. (B) Western blot analysis of PKA expression after treatment with $0.1-10 \mu \mathrm{M}$ LIN for $48 \mathrm{~h}$. (C) Cells were pretreated with the PKA inhibitor H89 $(2 \mu \mathrm{M})$ for $1 \mathrm{~h}$, and then $10 \mu \mathrm{M} \mathrm{LIN}$ was added for $48 \mathrm{~h}$. Western blot analysis showed that pretreatment with H89 counteracted the effect of LIN on BMP-2, p-SMAD1/5 and runt-related transcription factor 2 (RUNX2) expression. The quantitation of the blots was undertaken by densitometric analysis. Data are represented as the means $\pm \mathrm{SD}$ of three independent experiments. ${ }^{*} \mathrm{P}<0.05,{ }^{* * *} \mathrm{P}<0.01$ compared with the control; ${ }^{*} \mathrm{P}<0.01$ compared with LIN.

cell differentiation were investigated. BMPs are critical in regulating osteoblast differentiation and successive bone formation, and BMP-2, a BMP subfamily protein, has exhibited strong osteosynthetic activity in vivo and in vitro $(33,34)$. In the present study, noggin, a specific inhibitor of the BMP-2 signaling pathway, was used to block the activation of the BMP-2 pathway and osteogenic-related gene activity was then examined. The results showed that the inhibitory effect of noggin affected the expression of several osteogenic genes induced by LIN. These results suggested that the BMP-2 signaling pathway is involved in LIN-induced cell differentiation.
Serine/threonine kinase receptors and downstream transcription factors such as SMAD1/5 have previously been shown to mediate BMP-2 intracellular signals. After activation, SMAD1/5 is recognized and phosphorylated by type I receptors. This p-SMAD1/5 forms a complex with SMAD4 and then moves to the cell nucleus where it recognizes the regulatory elements of target genes associated with differentiation (35). In the present study, we noted that LIN enhanced BMP-2 production in MC3T3-E1 cells. In addition, the p-SMAD1/5 protein level was increased by LIN in MC3T3-E1 cells. These data suggest that BMP-2 mediates LIN-induced upregulation of differentiation in MC3T3-E1 cells. 

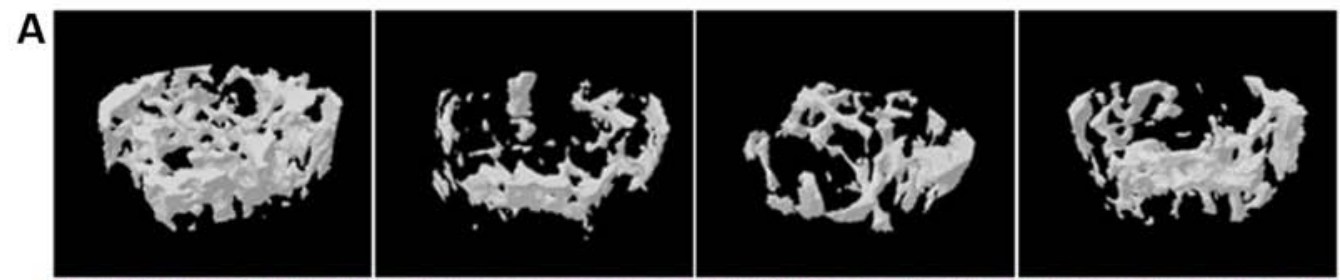

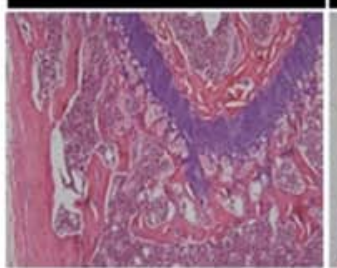

Sham

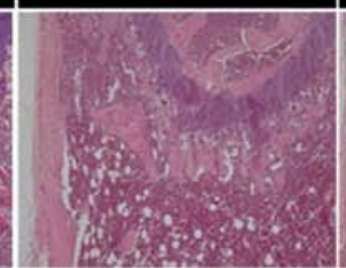

OVX

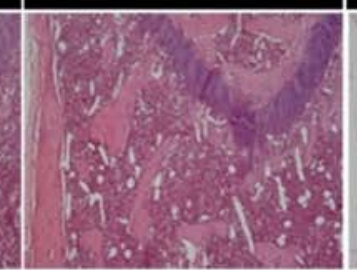

LIN (low)

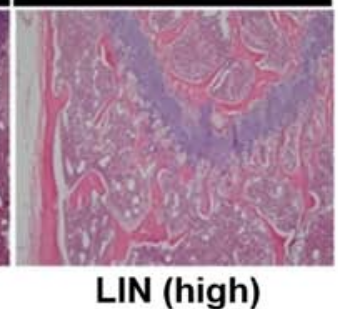

LIN (high)
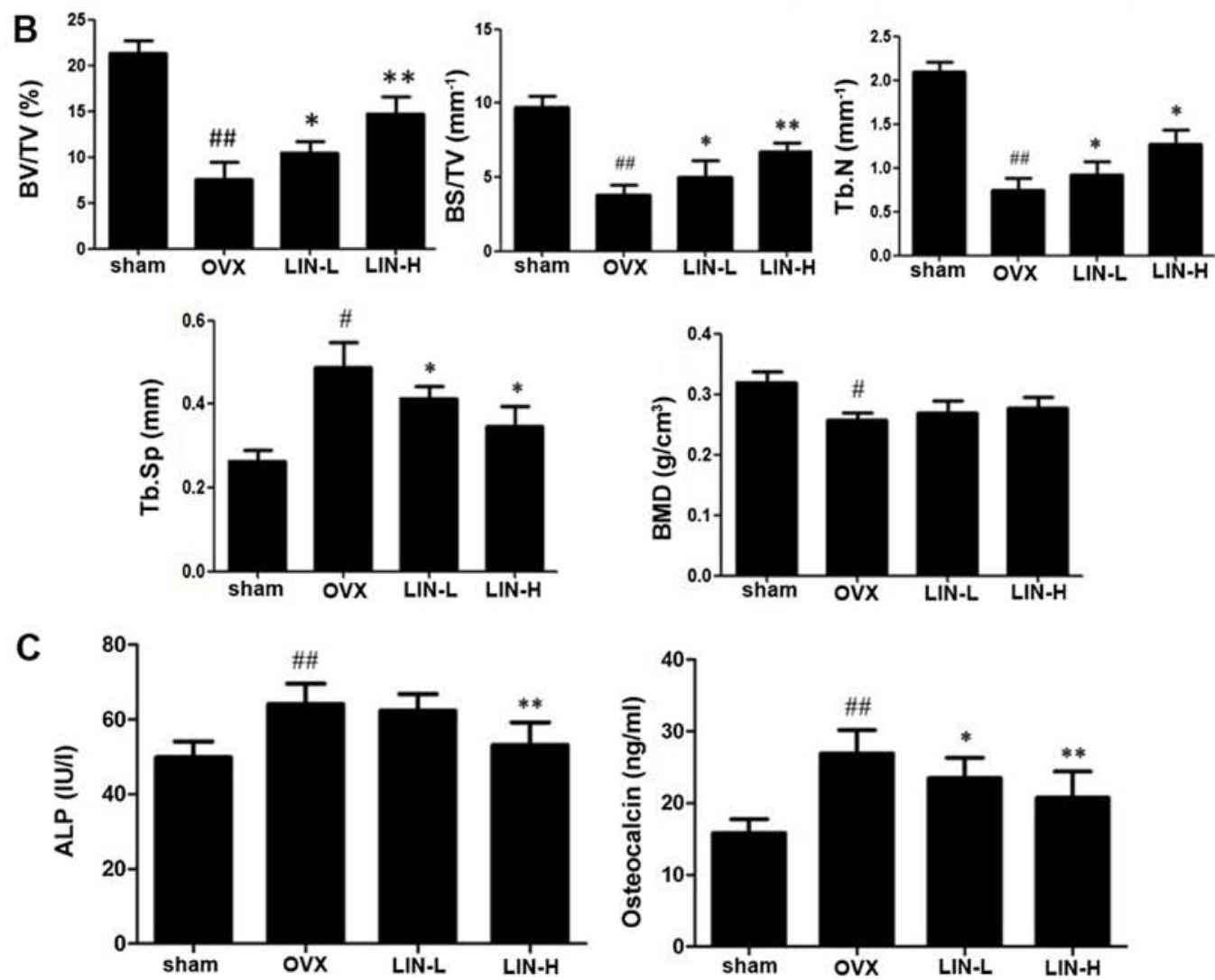

Figure 7. Effects of linarin (LIN) on bone loss in ovariectomized (OVX) mice. Ovariectomized mice were orally administered with LIN (50 or $150 \mathrm{mg} / \mathrm{kg}$ ) for 8 weeks. (A) Three-dimensional images of the proximal femurs were obtained with a $\mu \mathrm{CT}$. Femurs were fixed, decalcified, dehydrated, sectioned and H\&E stained (magnification, x10). (B) The microstructural indices were quantified from data obtained by the $\mu \mathrm{CT}$, including bone volume/tissue volume (BV/TV), bone surface/tissue volume (BS/TV), trabecular number (Tb.N), trabecular space (Tb.Sp) and bone mineral density (BMD). (C) Serum levels of alkaline phosphatase (ALP) and osteocalcin $(\mathrm{OCN})$ were analyzed with the respective kit. The results are expressed as the means $\pm \mathrm{SD}, \mathrm{n}=8-10 / \mathrm{group} .{ }^{*} \mathrm{P}<0.05,{ }^{* *} \mathrm{P}<0.01$ compared with OVX; ${ }^{\text {P }}<0.01$ compared with LIN; ${ }^{\#} \mathrm{P}<0.01$ compared with sham-operated group.

RUNX2, an important transcriptional factor for osteoblast differentiation, has been reported to be induced by BMP-2 (7,36). Moreover, RUNX2 directly interacts with the promoter regions of key osteoblast specific genes, including ALP, OCN and BSP (37). In the present study, the effect of LIN on RUNX2 expression in MC3T3-E1 cells was examined. The results showed that RUNX2 was activated by LIN at both mRNA and protein levels, indicating that LIN induces osteogenic differentiation via the BMP-2/RUNX2 pathway.

PKA, a cyclic adenosine monophosphate (cAMP)-dependent protein kinase, serves as a second messenger that is found ubiquitously in mammalian cells (38). Many compounds either directly or indirectly modulate PKA activity, and thereby promote the osteoblast differentiation of mesenchymal stem cells $(11,39,40)$. For example, recent reports have indicated that PKA regulates the osteogenic-related proteins such as RUNX2 and Osterix $(12,13)$. In another study, it has been shown that cAMP promotes SMAD-mediated BMP signaling via the PKA pathway (41). In the present study, we noted that PKA activation was induced by LIN in a time- and dose-dependent manner. These results were further confirmed by using a PKA inhibitor (H-89). LIN-induced BMP-2, SMAD1/5 and RUNX2 
protein expression was abrogated in the presence of $\mathrm{H}-89$. These results suggest that the LIN-induced osteoblast differentiation is mediated, at least in part, via the cAMP/PKA signaling pathway.

Several signaling pathways, including the PKA-, protein kinase $\mathrm{C}$-, mitogen-activated protein kinase-, and 5' AMP-activated protein kinase-dependent pathways have been shown to modulate osteoblast differentiation in response to external stimuli $(28,42,43)$. In the present study, co-treatment with $\mathrm{H}-89$ and LIN in MC3T3-E1 cells resulted in partial reversal of the activation of the BMP-2 signaling pathway. This indicated that PKA signaling is not the only pathway that modulates downstream of BMP-2 and enhances the osteogenic differentiation induced by LIN. The actual mechanism by which LIN regulates the osteoblast differentiation requires further investigation.

Finally, the in vivo effects of LIN on postmenopausal osteoporosis, which was stimulated by OVX-induced bone loss in mice, was investigated. The results showed that LIN (50 and $150 \mathrm{mg} / \mathrm{kg} /$ day) significantly improved the trabecular bone microarchitecture of distal femur in ovariectomized mice. This suggests that LIN is effective in preventing OVX-induced bone loss. These findings were also confirmed with histological examination of the femur tissues using H\&E staining. Due to imbalance between bone formation and resorption, the deterioration of bone microstructure with associated acceleration of bone turnover occurs (44). We noted that serum ALP and OCN levels were significantly increased in ovariectomized mice, which were considered to be markers of bone turnover. The treatment with LIN reversed the increase of osteoporotic markers in the mice which underwent OVX, indicating that LIN prevents bone loss through inhibition of bone turnover.

In conclusion, the present study indicated that LIN induces the differentiation and minezralization of MC3T3-E1 cells. The effects of LIN were mediated by activating BMP-2/RUNX2 pathway via PKA signaling and promoting osteoid gene expression. Moreover, LIN administration prevented OVX-induced deterioration of trabecular microarchitecture in vivo. In short, LIN should be considered as a natural alternative for postmenopausal osteoporosis.

\section{Acknowledgements}

We thank Professor Yao Sun for his excellent theoretical assistance. The present study was supported by the Natural Science Foundation of China (grant nos. 81371949 and 81470716), the Science and Technology commission of Shanghai, China (grant no. 13411951201) and the Shanghai Municipal Commission of Health and Family Planning (grant no. 2014065).

\section{References}

1. Ducy P, Schinke T and Karsenty G: The osteoblast: a sophisticated fibroblast under central surveillance. Science 289 1501-1504, 2000.

2. Teitelbaum SL: Bone resorption by osteoclasts. Science 289: 1504-1508, 2000.

3. Isenbarger DW and Chapin BL: Osteoporosis. Current pharmacologic options for prevention and treatment. Postgrad Med 101: 129-132, 136-137, 141-142, 1997.

4. Orija IB and Mehta A: Hormone replacement therapy: current controversies. Clin Endocrinol (Oxf) 59: 657, 2003.

5. Canalis E, Economides AN and Gazzerro E: Bone morphogenetic proteins, their antagonists, and the skeleton. Endocr Rev 24: 218-235, 2003.
6. Sykaras N and Opperman LA: Bone morphogenetic proteins (BMPs): how do they function and what can they offer the clinician? J Oral Sci 45: 57-73, 2003.

7. Phimphilai M, Zhao Z, Boules H, Roca H and Franceschi RT: BMP signaling is required for RUNX2-dependent induction of the osteoblast phenotype. J Bone Miner Res 21: 637-646, 2006.

8. Lee MH, Kwon TG, Park HS, Wozney JM and Ryoo HM: BMP-2-induced Osterix expression is mediated by Dlx 5 but is independent of Runx2. Biochem Biophys Res Commun 309: 689-694, 2003.

9. Pasqualucci L, Kitaura Y, Gu H and Dalla-Favera R: PKAmediated phosphorylation regulates the function of activationinduced deaminase (AID) in B cells. Proc Natl Acad Sci USA 103: 395-400, 2006.

10. Skalhegg BS and Tasken K: Specificity in the cAMP/PKA signaling pathway. Differential expression, regulation, and subcellular localization of subunits of PKA. Front Biosci 5: D678-D693, 2000.

11. Lo KW, Kan HM, Ashe KM and Laurencin CT: The small molecule PKA-specific cyclic AMP analogue as an inducer of osteoblast-like cells differentiation and mineralization. J Tissue Eng Regen Med 6: 40-48, 2012.

12. Li H, Jeong HM, Choi YH, Kim JH, Choi JK, Yeo CY, Jeong HG, Jeong TC, Chun C and Lee KY: Protein kinase a phosphorylates Dlx3 and regulates the function of Dlx3 during osteoblast differentiation. J Cell Biochem 115: 2004-2011, 2014.

13. He S, Choi YH, Choi JK, Yeo CY, Chun C and Lee KY: Protein kinase A regulates the osteogenic activity of Osterix. J Cell Biochem 115: 1808-1815, 2014.

14. Sassi AB, Harzallah-Skhiri F, Bourgougnon N and Aouni M: Antimicrobial activities of four Tunisian Chrysanthemum species. Indian J Med Res 127: 183-192, 2008.

15. Liu Q, Liu H, Yuan Z, Wei D and Ye Y: Evaluation of antioxidant activity of chrysanthemum extracts and tea beverages by gold nanoparticles-based assay. Colloids Surf B Biointerfaces 92: 348-352, 2012

16. He J, Chen F, Chen S, Lv G, Deng Y, Fang W, Liu Z, Guan Z and He C: Chrysanthemum leaf epidermal surface morphology and antioxidant and defense enzyme activity in response to aphid infestation. J Plant Physiol 168: 687-693, 2011.

17. Marongiu B, Piras A, Porcedda S, Tuveri E, Laconi S, Deidda D and Maxia A: Chemical and biological comparisons on supercritical extracts of Tanacetum cinerariifolium (Trevir) Sch. Bip. with three related species of chrysanthemums of Sardinia (Italy). Nat Prod Res 23: 190-199, 2009.

18. Kim YH, Lee YS and Choi EM: Linarin isolated from Buddleja officinalis prevents hydrogen peroxide-induced dysfunction in osteoblastic MC3T3-E1 cells. Cell Immunol 268: 112-116, 2011.

19. Suh KS, Rhee SY, Jung WW, Kim NJ, Jang YP, Kim HJ, Kim MK, Choi YK and Kim YS: Chrysanthemum zawadskii extract protects osteoblastic cells from highly reducing sugar-induced oxidative damage. Int J Mol Med 32: 241-250, 2013.

20. Han S, Sung KH, Yim D, Lee S, Lee CK, Ha NJ and Kim K: The effect of linarin on LPS-induced cytokine production and nitric oxide inhibition in murine macrophages cell line RAW264.7. Arch Pharm Res 25: 170-177, 2002.

21. Lou H, Fan P, Perez RG and Lou H: Neuroprotective effects of linarin through activation of the PI3K/Akt pathway in amyloid- $\beta$-induced neuronal cell death. Bioorg Med Chem 19: 4021-4027, 2011.

22. Qiaoshan Y, Suhong C, Minxia S, Wenjia M, Bo L and Guiyuan L: preparative purification of linarin extracts from Dendranthema indicum flowers and evaluation of its antihypertensive effect. Evid Based Complement Alternat Med 2014: 394276, 2014.

23. Li J, Zhang X, Yu Q, Fu X and Wang W: One-step separation of four flavonoids from Herba Salviae Plbeiae by HSCCC. J Chromatogr Sci 52: 1288-1293, 2014.

24. Yang RS, Lin WL, Chen YZ, Tang CH, Huang TH, Lu BY and Fu WM: Regulation by ultrasound treatment on the integrin expression and differentiation of osteoblasts. Bone 36: 276-283, 2005.

25. Wedemeyer C, Xu J, Neuerburg C, Landgraeber S, Malyar NM, von Knoch F, Gosheger G, von Knoch M, Löer F and Saxler G: Particle-induced osteolysis in three-dimensional micro-computed tomography. Calcif Tissue Int 81: 394-402, 2007.

26. Bellows CG, Aubin JE and Heersche JN: Initiation and progression of mineralization of bone nodules formed in vitro: the role of alkaline phosphatase and organic phosphate. Bone Miner 14: 27-40, 1991 
27. Hallahan AR, Pritchard JI, Chandraratna RA, Ellenbogen RG, Geyer JR, Overland RP, Strand AD, Tapscott SJ and Olson JM: BMP-2 mediates retinoid-induced apoptosis in medulloblastoma cells through a paracrine effect. Nat Med 9: 1033-1038, 2003.

28. Franceschi RT and Xiao G: Regulation of the osteoblast-specific transcription factor, Runx2: responsiveness to multiple signal transduction pathways. J Cell Biochem 88: 446-454, 2003.

29. Martin TJ and Sims NA: Osteoclast-derived activity in the coupling of bone formation to resorption. Trends Mol Med 11: 76-81, 2005.

30. Schilling T, Ebert R, Raaijmakers N, Schütze N and Jakob F: Effects of phytoestrogens and other plant-derived compounds on mesenchymal stem cells, bone maintenance and regeneration. J Steroid Biochem Mol Biol 139: 252-261, 2014.

31. Lian JB and Stein GS: Concepts of osteoblast growth and differentiation: basis for modulation of bone cell development and tissue formation. Crit Rev Oral Biol Med 3: 269-305, 1992.

32. Gordon JA, Tye CE, Sampaio AV, Underhill TM, Hunter GK and Goldberg HA: Bone sialoprotein expression enhances osteoblast differentiation and matrix mineralization in vitro. Bone 41 : 462-473, 2007.

33. Jia TL, Wang HZ, Xie LP, Wang XY and Zhang RQ: Daidzein enhances osteoblast growth that may be mediated by increased bone morphogenetic protein (BMP) production. Biochem Pharmacol 65: 709-715, 2003.

34. Takuwa Y, Ohse C, Wang EA, Wozney JM and Yamashita K: Bone morphogenetic protein-2 stimulates alkaline phosphatase activity and collagen synthesis in cultured osteoblastic cells, MC3T3-E1. Biochem Biophys Res Commun 174: 96-101, 1991.

35. Miyazono K: Signal transduction by bone morphogenetic protein receptors: functional roles of Smad proteins. Bone 25: 91-93, 1999.

36. Lian JB, Javed A, Zaidi SK, Lengner C, Montecino M, van Wijnen AJ, Stein JL and Stein GS: Regulatory controls for osteoblast growth and differentiation: role of Runx/Cbfa/AMl factors. Crit Rev Eukaryot Gene Expr 14: 1-41, 2004.
37. Ducy P, Zhang R, Geoffroy V, Ridall AL and Karsenty G: Osf2/Cbfa1: a transcriptional activator of osteoblast differentiation. Cell 89: 747-754, 1997.

38. Beavo JA and Brunton LL: Cyclic nucleotide research - still expanding after half a century. Nat Rev Mol Cell Biol 3: 710-718, 2002.

39. Wu X,Zeng LH, Taniguchi T and Xie QM: Activation of PKA and phosphorylation of sodium-dependent vitamin $\mathrm{C}$ transporter 2 by prostaglandin E2 promote osteoblast-like differentiation in MC3T3-E1 cells. Cell Death Differ 14: 1792-1801, 2007.

40. Siddappa R, Martens A, Doorn J, Leusink A, Olivo C, Licht R, van Rijn L, Gaspar C, Fodde R, Janssen F, et al: cAMP/PKA pathway activation in human mesenchymal stem cells in vitro results in robust bone formation in vivo. Proc Natl Acad Sci USA 105: 7281-7286, 2008.

41. Ohta Y, Nakagawa K, Imai Y, Katagiri T, Koike T and Takaoka K: Cyclic AMP enhances Smad-mediated BMP signaling through PKA-CREB pathway. J Bone Miner Metab 26: 478-484, 2008.

42. Xiao G, Jiang D, Thomas P, Benson MD, Guan K, Karsenty G and Franceschi RT: MAPK pathways activate and phosphorylate the osteoblast-specific transcription factor, Cbfa1. J Biol Chem 275: 4453-4459, 2000.

43. Kanazawa I, Yamaguchi T, Yano S, Yamauchi M, Yamamoto M and Sugimoto T: Adiponectin and AMP kinase activator stimulate proliferation, differentiation, and mineralization of osteoblastic MC3T3-E1 cells. BMC Cell Biol 8: 51, 2007.

44. Turner RT, Vandersteenhoven JJ and Bell NH: The effects of ovariectomy and 17 beta-estradiol on cortical bone histomorphometry in growing rats. J Bone Miner Res 2: 115-122, 1987. 